\title{
Mindsets of Early-Career Family Physicians Trained in Competency-Based Education
}

\author{
Mao Ding, MA | Sudha Koppula, MD, MCISc | Olga Szafran, MHSA | Lillian Au, MD | Oksana \\ Babenko, PhD
}

PRIMER. 2021;5:39.

Published: 10/19/2021 | DOI: 10.22454/PRiMER.2021.389603

\section{Abstract}

Introduction: The objective of this study was to examine the mindsets (mastery, performance approach, performance avoidance) of early-career family physicians following graduation from a competency-based education residency program.

Methods: This was a longitudinal, cohort, survey study of family medicine residents at a large Canadian university. The 2015-2017 cohort of family medicine residents was surveyed at three time points: (1) at the end of residency training; (2) at 1 year in clinical practice; and (3) at 3 years in clinical practice. We used Baranik et al's instrument to measure three types of mindsets. We performed descriptive and multivariate analyses using SPSS 26.0 software.

Results: Irrespective of the time in practice, mean scores were the highest on the mastery mindset and the lowest on the performance avoidance mindset measures $(P<.001)$. Over time, the mastery mindset scores tended to decrease $(P=.04)$.

Conclusion: Family physicians trained in competency-based education continued to be mastery-oriented in the first 3 years of clinical practice. This finding is reassuring given that the mastery mindset is associated with professional well-being and long-term success. Nonetheless, because mastery mindset scores appeared to decrease over time, residency programs need to ensure graduating residents are equipped with knowledge and tools to remain mastery-oriented throughout the course of their professional careers.

\section{Introduction}

Physicians must keep current with evolving medical evidence and guidelines to be able to respond to patients' changing needs. Early-career physicians encounter additional pressures in the steep learning curve of independent practice. Hence, a mastery mindset, defined as "a collection of beliefs and attitudes that result in a self-directed, persistent, and intrinsically motivated drive for knowledge, ${ }^{1,2}$ is required for lifelong learning following medical school and residency.,4

The high stakes of medical practice and the associated fear of making a poor clinical decision can ensue a mindset of performance or demonstration of competence for purposes of impression management 
(performance approach mindset) or ego-protection (performance avoidance mindset). ${ }^{5}$ Empirical research indicates that in general populations, performance mindsets are associated with many undesirable outcomes, including psychological distress, procrastination, cheating, and surface learning. ${ }^{6-11}$ In contrast, mastery mindset supports psychological well-being, engagement, perseverance, and effective response to challenges ${ }^{6-8}$, 11-13; it is needed for engaging in lifelong learning, beyond one's formal education. 3, 4, 14

Results of our previous research with family medicine (FM) residents indicate that competency-based medical education (CBME) ${ }^{15,16}$ has the potential to foster a mindset of mastery in learners. ${ }^{5} \mathrm{CBME}$ is an educational approach to training that places less emphasis on static medical knowledge and greater emphasis on the range of competencies required to be a skilled physician (eg, communication, professionalism, lifelong learning). ${ }^{17,18}$

Although the results of the earlier research are reassuring, it is unknown whether graduating residents continue to be mastery-oriented beyond residency training in their independent clinical practice. As such, the objective of this longitudinal, cohort study was to examine the mindsets of early-career family physicians at the end of competency-based residency training and at 1 and 3 years in clinical practice.

\section{Methods}

This was a longitudinal survey study of the 2015-2017 FM resident cohort $\left(n=70 ; 57 \%\right.$ female; $M_{\text {age }}=29$ years, $\mathrm{SD}_{\text {age }}=4.4$ years) at the University of Alberta, Canada. Our two-year FM residency program transitioned to competency-based education in $2009 .{ }^{19} \mathrm{It}$ is grounded in the best practices of formative feedback and guided self-assessment ${ }^{20}$ to encourage residents to take an active, self-directed role in their education.

To examine mindsets in the cohort of early-career family physicians over time, we undertook data collection at three time points: (1) at the end of residency training (June 2017); (2) at 1 year in clinical practice (July 2018August 2018); and (3) at 3 years in clinical practice (July 2020-August 2020). We obtained ethics approval from the institutional research ethics board prior to data collection. Taking part in the study was voluntary. Consent was implied by the return of a completed questionnaire. Each participant was assigned a study identification number to allow linking of responses over time.

To measure the three types of mindsets (mastery, performance approach, performance avoidance), we used the Baranik et al's instrument. ${ }^{21}$ Sample mindset items included "I am willing to take on challenging work assignments that I can learn from" (mastery mindset, four items); "I prefer to work on tasks where I can prove my competence to others" (performance approach mindset, four items); and "I prefer to avoid situations at work where I might perform poorly" (performance avoidance mindset, four items). Participants were asked to indicate their level of agreement with each statement ( $1=$ not at all agree; $10=$ completely agree).

Using SPSS 26.0 software, we first performed descriptive analyses (means, standard deviations) and examined trends in the data. Higher average (mean) mindset scores were indicative of endorsing a respective mindset to a greater extent. To test for significance in the observed means on the three mindset measures, we performed multivariate analysis of variance (MANOVA), which is appropriate when multiple dependent variables are considered simultaneously.

\section{Results}

Of 70 eligible residents, 52 (74\%), 43 (61\%), and 29 (41\%) completed the questionnaire at each of the three data collection points, respectively. Descriptive statistics for the three data collection points are shown in Table 1. Irrespective of the time in clinical practice, mean scores were the highest on the mastery mindset and the 
lowest on the performance avoidance mindset measures (MANOVA; $P<.001$ ). Over time, however, the mastery mindset scores tended to decrease; this downward trend was statistically significant $(P=.04)$. Figure 1 shows observed trends in the data.

\section{Discussion}

Both research and the understanding of mindsets along the medical education continuum are nascent. What is known is that traditional, non-CBME, approaches to medical education tend to reinforce the mindset of performance or "looking good." 3 This is a particular concern in traditional undergraduate medical education where learners strive for the highest possible grade and ranking. ${ }^{3} \mathrm{It}$ is possible that the mindset of performance remains dominant in learners as they begin residency training. In recent years, many residency programs have transitioned or are in the process of transitioning to CBME. As such, an understanding of what mindsets CBME fosters in learners in the long run is important for developing strategies for lifelong learning.

Our key study finding is that, upon completion of the competency-based education residency program, family physicians continued to be mastery-oriented in the first three years of independent clinical practice. This is not unexpected, given that coaching of residents in clinical settings is the cornerstone of our FM residency program. ${ }^{19}$ Clinical preceptors observe residents performing specific competencies on a daily basis and provide actionable, growth-oriented feedback on their progress toward competence, thus fostering the mastery mindset in residents.

Mastery mindset scores, however, tended to decrease gradually over time. There is robust literature on how different desirable traits (eg, empathy, idealism) ${ }^{22-24}$ decline over time and how undesirable outcomes (eg, depression, burnout ${ }^{25-28}$ increase over the trajectory of medical training. With respect to the observed decline in mastery mindset scores, we speculate that it speaks to the pressures early-career physicians face as they transition from residency training to independent clinical practice. During this transition, physicians are confronted with decisions related to location of practice, business aspects of practice, as well as the transition from supervised to independent practice, all of which may have contributed to the observed decline of the mastery mindset in the first 3 years. Given the documented benefits of the mastery mindset for professional well-being and long-term success, ${ }^{6-8,11-13}$ residency programs need to ensure that their graduates are equipped with explicit knowledge and tools to help them remain mastery-oriented throughout the course of their careers. This can be achieved through the provision of educational sessions and facilitated transition-to-practice groups during residency training. A learning community, such as a journal club ${ }^{29}$ consisting of residents and clinicians, can also promote mastery and lifelong learning and foster a genuine learning culture through engagement in peer-to-peer learning. ${ }^{30}$ This type of community and learning culture can be supported from the beginning of residency training. Finally, irrespective of the career stage, involvement in teaching has been shown to be one of the best means for physicians to support their lifelong learning strategy and remain mastery-oriented. ${ }^{30,31}$

A strength of this study is the collection of repeated longitudinal data from one cohort of family medicine residents, which facilitated the analysis of trends over time. However, the observed trends in the data should be considered in light of the declining numbers in returned questionnaires over time, which is a known challenge in longitudinal studies. ${ }^{32}$ It could be that predominantly mastery-oriented individuals continued to participate in the study over time. Additionally, the scheduled third data collection coincided with the COVID-19 pandemic, negatively impacting the response rate. To encourage greater participation as well as to keep the survey short, we did not ask respondents to provide demographic information such as age and gender. Following only one resident cohort also limited the sample size. The findings may not be generalizable to family medicine resident cohorts with a different demographic profile and/or those who completed a residency program longer than 2 years and/or those not trained in CBME. To improve generalizability, future studies should consider following more than one cohort and including residents from different programs and/or locations. 


\section{Tables and Figures}

Table 1: Mindset Scores of Early-Career Family Physicians at Three Time Points

\begin{tabular}{|l|c|c|c|}
\hline \multirow{2}{*}{ Mindset Type } & \multicolumn{3}{|c|}{$\begin{array}{c}\text { Mindset Scores at Three Time Points } \\
\text { Mean (SD) }\end{array}$} \\
\hline Mastery & $\begin{array}{c}\text { End of Residency } \\
\text { Training } \\
\mathbf{n = 5 2}\end{array}$ & $\begin{array}{c}\text { 1 Year Into Clinical } \\
\text { Practice } \\
\mathbf{n = 4 3}\end{array}$ & $\begin{array}{c}\text { 3 Years Into } \\
\text { Clinical Practice } \\
\mathbf{n = 2 9}\end{array}$ \\
\hline Performance approach & $\mathbf{7 . 9 0 ( 1 . 0 0 )}$ & $\mathbf{7 . 6 4}(\mathbf{1 . 1 4})$ & $\mathbf{7 . 5 2 ( 0 . 8 7 )}$ \\
\hline Performance avoidance & $5.96(1.78)$ & $5.63(1.60)$ & $5.72(1.54)$ \\
\hline
\end{tabular}

The mean differences between mastery and performance avoidance scores over time were significant $(P<.001)$. The downward trend in mastery scores, shown in bold, was significant $(P=.04)$.

Figure 1: Trends in Mindset Scores of Early-Career Family Physicians at Three Time Points (Mean Scores Are Shown in Circles)

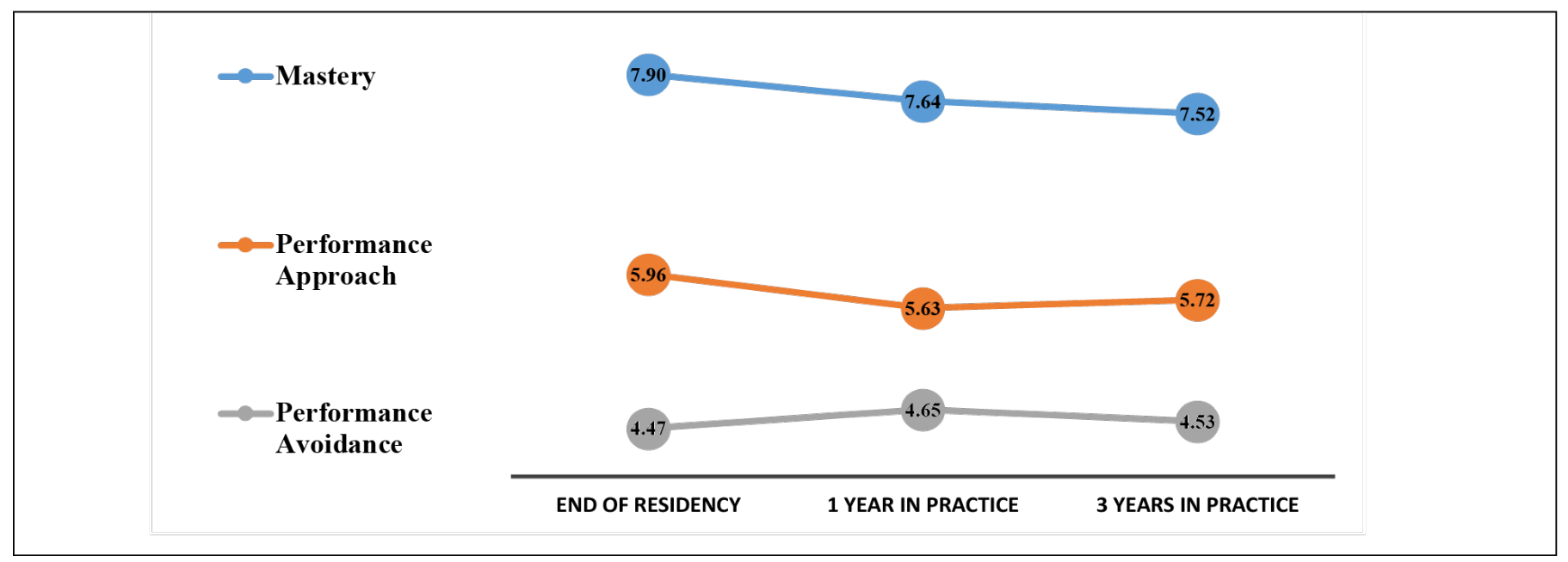

\section{Acknowledgments}

Financial support: This work was supported by funding from the Social Sciences and Humanities Research Council (grant \#430-2016-00267) and the Northern Alberta Academic Family Physicians Fund.

\section{Corresponding Author}

Oksana Babenko, PhD

Department of Family Medicine, 6-10 University Terrace, University of Alberta, Edmonton, Alberta, Canada T6G 2T4. 1-780-248-1729. Fax: 1-780-492-2593.

oksana.babenko@ualberta.ca

\section{Author Affiliations}

Mao Ding, MA - Department of Family Medicine, University of Alberta, Edmonton, Canada Sudha Koppula, MD, MCISc - Department of Family Medicine, University of Alberta, Edmonton, Canada Olga Szafran, MHSA - Department of Family Medicine, University of Alberta, Edmonton, Canada Lillian Au, MD - Department of Family Medicine, University of Alberta, Edmonton, Canada 
Oksana Babenko, PhD - Department of Family Medicine, University of Alberta, Edmonton, Canada

\section{References}

1. Dweck CS. Mindset: The New Psychology of Success. New York: Ballantine Books; 2006.

2. Sloychuk J, Szafran O, Duerksen K, Babenko O. Association between family medicine residents' mindsets and in-training exam scores. PRiMER Peer-Rev Rep Med Educ Res. 2020;4:33. doi:10.22454/PRiMER.2020.796230

3. Richardson D, Kinnear B, Hauer KE, et al; ICBME Collaborators. Growth mindset in competency-based medical education. Med Teach. 2021;43(7):751-757; Epub ahead of print. doi:10.1080/0142159X.2021.1928036

4. Teunissen PW, Bok HGJ. Believing is seeing: how people's beliefs influence goals, emotions and behaviour. Med Educ. 2013;47(11):1064-1072. doi:10.1111/medu.12228

5. Babenko O, Szafran O, Koppula S, Au L. Motivations for learning of family medicine residents trained in competency-based education. Educ Prim Care. 2018;29(2):86-93. doi:10.1080/14739879.2017.1362666

6. Elliot AJ, Hulleman CS. Achievement goals. In: Elliot AJ, Dweck CS, Yeager DS, eds. Handbook of Competence and Motivation: Theory and Application. 2nd ed. New York: Guilford Press; 2017:43-60.

7. Dweck CS, Molden DC. Mindsets: their impact on competence motivation and acquisition. In: Elliot AJ, Dweck CS, Yeager DS, eds. Handbook of Competence and Motivation: Theory and Application. 2nd ed. New York: Guilford Press; 2017:135-154.

8. Grant H, Dweck CS. Clarifying achievement goals and their impact. J Pers Soc Psychol. 2003;85(3):541-553. doi:10.1037/0022-3514.85.3.541

9. Howell CS, Watson DC. Procrastination: associations with achievement goal orientation and learning strategies. Pers Individ Dif. 2007;43(1):167-178. doi:10.1016/j.paid.2006.11.017

10. Van Yperen NW, Hamstra MR, Van der Klauw M. To win, or not to lose, at any cost: the impact of achievement goals on cheating. Br J Manage. 2011;22:S5-S15. doi:10.1111/j.1467-8551.2010.00702.x

11. Miller AL, Fassett KT, Palmer DL. Achievement goal orientation: a predictor of student engagement in higher education. Motiv Emot. 2021;45(3):327-344. doi:10.1007/s11031-021-09881-7

12. Dupeyrat $C$, Mariné $C$. Implicit theories of intelligence, goal orientation, cognitive engagement, and achievement: a test of Dweck's model with returning to school adults. Contemp Educ Psychol. 2005;30(1):43-59. doi:10.1016/j.cedpsych.2004.01.007

13. Yeager DS, Dweck CS. Mindsets that promote resilience: when students believe that personal characteristics can be developed. Educ Psychol. 2012;47(4):302-314.

doi:10.1080/00461520.2012.722805

14. Babenko O, Daniels LM, Ross S, White J, Oswald A. Medical student well-being and lifelong learning: $A$ motivational perspective. Educ Health (Abingdon). 2019;32(1):25-32. doi:10.4103/efh.EfH_237_17

15. Frank JR, Snell LS, Cate OT, et al. Competency-based medical education: theory to practice. Med Teach. 2010;32(8):638-645. doi:10.3109/0142159X.2010.501190

16. Frank JR, Snell L, Englander R, Holmboe ES, Collaborators ICBME; ICBME Collaborators. Implementing competency-based medical education: moving forward. Med Teach. 2017;39(6):568-573. doi:10.1080/0142159X.2017.1315069

17. Tannenbaum D, Kerr J, Konkin J, et al. Triple C competency-based curriculum. Report of the Working Group on Postgraduate Curriculum Review - Part 1. Mississauga, ON: College of Family Physicians of Canada; 2011. Accessed October 5, 2021. https://www.cfpc.ca/CFPC/media/Resources/Education /WGCR_TripleC_Report_English_Final_18Mar11.pdf.

18. Oandasan I, Saucier D, eds. Triple C Competency-based Curriculum Report - Part 2: Advancing Implementation. Mississauga, ON: College of Family Physicians of Canada; 2013. Accessed October 5, 2021. www.cfpc.ca/uploadedFiles/Education/_PDFs/TripleC_Report_pt2.pdf 
19. Ross S, Poth CN, Donoff M, et al. Competency-based achievement system: using formative feedback to teach and assess family medicine residents' skills. Can Fam Physician. 2011;57(9):e323-e330.

20. Babenko O, Campbell-Scherer D, Schipper S, et al. Examining accuracy of self-assessment of In-Training Examination performance in a context of guided self-assessment. Fam Med. 2017;49(6):451-455.

21. Baranik LE, Barron KE, Finney SJ. Measuring goal orientation in a work domain: construct validity evidence for the 2 × 2 framework. Educ Psychol Meas. 2007;67(4):697-718. doi:10.1177/0013164406292090

22. Neumann M, Edelhäuser F, Tauschel D, et al. Empathy decline and its reasons: a systematic review of studies with medical students and residents. Acad Med. 2011;86(8):996-1009.

doi:10.1097/ACM.0b013e318221e615

23. Chen DCR, Kirshenbaum DS, Yan J, Kirshenbaum E, Aseltine RH. Characterizing changes in student empathy throughout medical school. Med Teach. 2012;34(4):305-311.

doi:10.3109/0142159X.2012.644600

24. Mader EM, Roseamelia C, Morley CP. The temporal decline of idealism in two cohorts of medical students at one institution. BMC Med Educ. 2014;14(1):58. doi:10.1186/1472-6920-14-58

25. Mata DA, Ramos MA, Bansal N, et al. Prevalence of depression and depressive symptoms among resident physicians: a systematic review and meta-analysis. JAMA. 2015;314(22):2373-2383. doi:10.1001/jama.2015.15845

26. Rotenstein LS, Ramos MA, Torre M, et al. Prevalence of depression, depressive symptoms, and suicidal ideation among medical students: a systematic review and meta-analysis. JAMA. 2016;316(21):2214-2236. doi:10.1001/jama.2016.17324

27. Dyrbye LN, West CP, Satele D, et al. Burnout among U.S. medical students, residents, and early career physicians relative to the general U.S. population. Acad Med. 2014;89(3):443-451. doi:10.1097/ACM.0000000000000134

28. Hansell MW, Ungerleider RM, Brooks CA, Knudson MP, Kirk JK, Ungerleider JD. Temporal trends in medical student burnout. Fam Med. 2019;51(5):399-404. doi:10.22454/FamMed.2019.270753

29. Lee AG, Boldt HC, Golnik KC, et al. Using the Journal Club to teach and assess competence in practicebased learning and improvement: a literature review and recommendation for implementation. Surv Ophthalmol. 2005;50(6):542-548. doi:10.1016/j.survophthal.2005.08.002

30. Murthy VK, O’Brien B, Dhaliwal G. An inquiry into the early careers of master clinicians. J Grad Med Educ. 2018;10(5):500-506. doi:10.4300/JGME-D-18-00208.1

31. Ding M, Babenko O, Koppula S, Oswald A, White J. Physicians as teachers and lifelong learners. J Contin Educ Health Prof. 2019;39(1):2-6. doi:10.1097/CEH.0000000000000228

32. Phillips AW, Friedman BT, Utrankar A, Ta AQ, Reddy ST, Durning SJ. Surveys of health professions trainees: prevalence, response rates, and predictive factors to guide researchers. Acad Med. 2017;92(2):222-228. doi:10.1097/ACM.0000000000001334

Copyright $@ 2021$ by the Society of Teachers of Family Medicine 